\title{
The somatic marker theory in the context of addiction: contributions to understanding development and maintenance
}

This article was published in the following Dove Press journal:

Psychology Research and Behavior Management

6 July 2015

Number of times this article has been viewed

\author{
Vegard V Olsen' \\ Ricardo G Lugo' \\ Stefan Sütterlin ${ }^{1,2}$ \\ 'Section of Psychology, Lillehammer \\ University College, Lillehammer, \\ ${ }^{2}$ Department of Psychosomatic \\ Medicine, Division of Surgery \\ and Clinical Neuroscience, Oslo \\ University Hospital - Rikshospitalet, \\ Oslo, Norway
}

\begin{abstract}
Recent theoretical accounts of addiction have acknowledged that addiction to substances and behaviors share inherent similarities (eg, insensitivity to future consequences and self-regulatory deficits). This recognition is corroborated by inquiries into the neurobiological correlates of addiction, which has indicated that different manifestations of addictive pathology share common neural mechanisms. This review of the literature will explore the feasibility of the somatic marker hypothesis as a unifying explanatory framework of the decision-making deficits that are believed to be involved in addiction development and maintenance. The somatic marker hypothesis provides a neuroanatomical and cognitive framework of decision making, which posits that decisional processes are biased toward long-term prospects by emotional marker signals engendered by a neuronal architecture comprising both cortical and subcortical circuits. Addicts display markedly impulsive and compulsive behavioral patterns that might be understood as manifestations of decision-making processes that fail to take into account the long-term consequences of actions. Evidence demonstrates that substance dependence, pathological gambling, and Internet addiction are characterized by structural and functional abnormalities in neural regions, as outlined by the somatic marker hypothesis. Furthermore, both substance dependents and behavioral addicts show similar impairments on a measure of decision making that is sensitive to somatic marker functioning. The decision-making deficits that characterize addiction might exist a priori to addiction development; however, they may be worsened by ingestion of substances with neurotoxic properties. It is concluded that the somatic marker model of addiction contributes a plausible account of the underlying neurobiology of decision-making deficits in addictive disorders that is supported by the current neuroimaging and behavioral evidence. Implications for future research are outlined.
\end{abstract}

Keywords: addiction, somatic marker hypothesis, decision making, emotion, Iowa Gambling Task

\section{Introduction}

Addiction is characterized as a condition in which neural systems involved in motivation and behavioral control promote self-regulatory failure that persists in the face of increasing negative consequences. ${ }^{1}$ The component model of addiction posits that the addiction state involves increased salience attribution to the addictive object, mood modification, development of tolerance, withdrawal, internal and external conflict, and relapse. ${ }^{2}$ This conceptualization implies that addiction is not confined to chemical substances, but can also involve behaviors like excessive gambling and Internet use. Recognition of the behavioral similarities across addictions coincides with increasing evidence indicating that chemical and nonchemical addictions might share common neural mechanisms. ${ }^{3-5}$ Impulsive and compulsive behavior in addiction
Correspondence: Stefan Sütterlin Section of Psychology, Lillehammer University College, Box 952, 2604

Lillehammer, Norway

Tel +4761288158

Email stefan.sutterlin@hil.no 
have been associated with defective decision making. ${ }^{6}$ A better understanding of the abnormal decisional processes observed in various kinds of addiction has therefore come into focus of research on development and maintenance of addiction. The somatic marker hypothesis (SMH) provides a theoretical framework for an explanation of dysfunctional decision-making patterns in addicts. This review provides an overview on the current state of research on decision making in addiction with a particular focus on the role of somatic marker theory's contributions.

The decision-making deficits in addiction are comparable to those seen in patients with prefrontal cortical lesions - a marked obliviousness to future consequences of decisions and deficient experience-based learning. ${ }^{7}$ This observation and supporting physiological and anatomical assessments have led to the notion that pathophysiology in the prefrontal cortex could be an important neural underpinning of addiction. ${ }^{8-10}$ Decisional outcomes following affective-cognitive integration in prefrontal areas are influenced by afferent connections to limbic system areas. The SMH provides a system-level framework describing how decision-making processes are influenced by emotional signals arising from bioregulatory changes that express themselves in both brain and body. ${ }^{11,12}$ The theory developed from studies of the decision-making deficits of patients with lesions in the ventromedial prefrontal cortex (vmPFC). ${ }^{13,14}$ The SMH echoes and extends the Jamesian account of peripheral feedback, positing that emotional biasing signals from the periphery guide the decision-making process toward long-term prospects in situations characterized by complexity and uncertainty. ${ }^{15}$ Evidence indicates that the normal operation of somatic markers is dependent upon various structures involved in expressing emotions in the periphery, such as the vmPFC and amygdala, as well as structures implicated in the central representation of the changes taking place in the body proper (somatosensory cortex, insular cortex, basal ganglia, cingulate cortex). ${ }^{16-18}$

The empirical support for the SMH has largely been derived from an affective decision-making paradigm aimed at mimicking real life decision making in the way it factors uncertainty, reward, and punishment - the Iowa Gambling Task (IGT). ${ }^{11}$ Bechara et al ${ }^{19}$ suggested a correlation between successful performance and heightened skin conductance responses (SCR) anticipating unconscious disadvantageous decisions on this task. ${ }^{19-21}$ These anticipative SCRs have been interpreted as an index of somatic marker signals, and are absent in patients with lesions in the vmPFC. Interestingly, the paradigm has been used to explore decision making in various clinical populations and several studies indicate that somatic marker deficits might underlie a plethora of clinical manifestations of impaired decision making, including those seen in drug addiction, ${ }^{22}$ psychopathy, ${ }^{23,24}$ anxiety, ${ }^{25}$ obsessive-compulsive disorder, ${ }^{26}$ and panic disorder. ${ }^{27}$

Increasing evidence indicates that addiction is characterized by a defect in the somatic marker mechanism that normally supports the selection of adaptive behavior, giving rise to inappropriate emotional markers of the anticipated negative consequences of future action, thus promoting selfregulatory failure. ${ }^{10,28}$ Previous literature reviews exploring the relationship between somatic markers and addiction have implied that the SMH can explain dysfunctional decision making in both substance and behavioral forms of addiction,

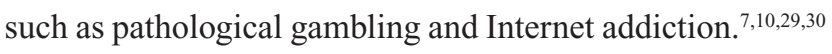
While previous literature reviewed findings supporting the model's ability to explain dysfunctional decision making in drug addiction, it does not provide conclusive evidence for whether its predictions hold true for behavioral addictions as well. Moreover, the etiology of the somatic marker defects has not been elaborated upon; further research is required to determine whether the defective somatic marker signaling system thought to be involved in addiction is a premorbid vulnerability factor or a result of addictive behavior.

First, a brief overview of the current understanding of the neurobiology of somatic marker signaling will be provided. Thereafter, the neurophysiological and neurocognitive evidence pertinent to predictions from the somatic marker model of addiction will be reviewed, and the model's applicability to defective decision making in addiction will be critically appraised. The scope will be limited to drug addiction, pathological gambling, and Internet addiction, as these represent the addictive disorders that have received the most research attention. Finally, the review will discuss the etiology of somatic marker defects and the possibility of a diathetic vulnerability for addiction from the defective decision making that results from a dysfunctional somatic marker signaling system.

\section{Neurobiological correlates of somatic marker signaling}

The SMH posits that the neural embodiment of somatic state information in relation to situational contingencies holds biasing properties capable of guiding the decision-making process via reactivation of somatic states previously paired with an option-outcome pair (overviews have been previously published $^{15,16}$ ). The SMH distinguishes between two different triggers of somatic state activation, each associated with distinct neural substrates. ${ }^{31}$ Primary inducers refer to innate 
or learned stimuli that are associated with automatic somatic (emotional) responses, mediated by subcortical structures involved in emotional processing, where the amygdala is an essential structure. Secondary inducers refer to cognitive stimuli generated from thoughts and memories of an actual or hypothetical emotional event - eg, the memory of taking a drug or the thoughts about taking the drug in the future. While operating via the same somatic effector structures in the brain stem and hypothalamic area that the amygdala does in the case of primary induction, induction from secondary inducers is associated with higher-order associative areas in the vmPFC, which are capable of encoding and reactivating somatic states associated with a particular option-outcome pair. ${ }^{16}$ Moreover, once a dispositional representation is established, thus allowing secondary induction, the reactivation may proceed as an intracerebral replay of the changes in the soma, via the as-if loop mechanism that bypasses the body proper altogether. ${ }^{32}$

Both the vmPFC and the amygdala are richly connected to somatic effector structures in hypothalamic and brain stem nuclei that are capable of playing out bioregulatory changes in the body proper - eg, they initiate a somatic marker. These emotional changes are engendered in the body via the spinal cord, cranial nerves, and endocrine signaling. The afferent projections of the ensuing changes are represented in somatotopically-organized regions. The somatosensory cortices in the parietal lobe (SI and SII) and the insular cortices monitor interoceptive information continuously, ${ }^{33}$ and especially the anterior part of the insular cortex is believed to be a key neural underpinning for the conscious experience of the body and the emotions of which it serves as a theater. ${ }^{18,34}$ Indeed, neuroimaging studies have shown that the strength of insular activity correlates with both perceived emotional intensity and accuracy in interoceptive judgments. ${ }^{18,35}$ Moreover, various studies have demonstrated associations between insular activation and decisional outcomes. For example, Werner et $\mathrm{al}^{36}$ reported that insular activation is predictive of successful intuitive decision making. Related, high interoceptive accuracy is positively associated with higher susceptibility to emotional biases in an emotionally framed decision-making task ${ }^{37}$ and with detrimental effects of dysfunctionally processed interoceptive biases on decision making in patients with panic disorder. ${ }^{27}$ These findings and strong evidence on the neuronal correlates of interoceptive accuracy identified in the anterior insular cortex ${ }^{18}$ strengthen previous suggestions that this region is an integral structure for the behavioral effects of somatic markers. The role of the insular cortex in somatic marker biasing actions is fur- ther corroborated by lesion studies linking damage to this structure to distinct decision-making deficits, especially an insensitivity to value differences among response options. ${ }^{38,39}$ Thus, impaired functioning of the insular cortex may reduce the individual's ability to determine the value of response options due to inefficient utilization of peripheral changes that options of emotional salience typically induce.

The biasing actions of somatic markers are not restricted to the immediate selection of adaptive behavioral programs. Somatic markers are hypothesized to assist overt reasoning processes where they amplify some response options over others, in such a way that more executive resources are dedicated to these options. ${ }^{16}$ This assumption is consistent with the elaborate connections between regions of the somatic marker system, especially the vmPFC, and regions involved in working memory and conflict resolution, such as the dorsolateral prefrontal cortex (dlPFC) and the anterior cingulate cortex. ${ }^{40,41}$ Moreover, studies employing executive load paradigms combined with tasks measuring decision making suggest that regions involved in mediating executive resources, particularly working memory, are necessary, but not sufficient, for the overt guidance of behavior through somatic marker biasing actions. ${ }^{42,43}$ Furthermore, it is assumed that somatic markers can bias behavior implicitlythat is, outside conscious awareness - through connections to regions in the basal ganglia, especially the striatum. ${ }^{10}$ This is of special interest in the context of addiction, with compelling evidence suggesting that increased dopamine transmission from the mesolimbic dopamine system to the ventral striatum drives incentive motivational processes outside its adaptive range in addiction, resulting in an attentional bias toward and increased wanting of the desired object. ${ }^{44,45}$ Thus, there may be an interaction at the level of the striatum between dopamine-driven processes involved in incentive motivation and affective-cognitive signals from prefrontal cortical circuits.

\section{Neurochemical mediation of somatic state influence on behavior and cognition}

Advances in neuropharmacological research have begun to unravel how neurochemical transmitter substances influence behavior and cognition. Particularly, the monoamines have received considerable attention and are believed to play important roles in various cognitive processes, including decision making. ${ }^{46}$ The monoamine neurotransmitters have also been a major focus in the study of psychopathology, including addiction, ${ }^{47}$ and substantial evidence favors a 
causal role for these neurochemicals in many psychopathological conditions. While the precise role of these neuromodulating substances in decision-making processes remains unknown, there is evidence to indicate that the biasing properties of somatic markers are due, in large part, to changes in the release of these transmitter substances in various parts of the brain implicated in cognitive and emotional processing - eg, the vmPFC, amygdala, insular cortex, and striatum. ${ }^{16}$

Accumulating evidence suggests that the serotonergic system is an integral part of adaptive decision making and might play a central role in the biasing properties of somatic markers. ${ }^{48,49}$ Rogers et $\mathrm{al}^{50}$ found evidence of an association between low levels of serotonin (5-HT), induced by dietary challenge, and poor performance on a paradigm sensitive to orbitofrontal/vmPFC functioning. Moreover, low 5-HT levels have been consistently associated with an increased tendency toward temporal discounting ${ }^{51,52}$ and impulsive behavior, ${ }^{53}$ both of which are clearly involved in addictive behavior. The dopaminergic system has also been implicated in affective decision making, with evidence demonstrating an association between lowered levels of dopamine and impaired performance on the IGT. ${ }^{54}$ Specifically, reduced levels of dopamine seem to interfere with performance in the first part of the task, when the decision-making process is guided by implicit knowledge of the task contingencies. In contrast, it has been found that manipulations of the serotonergic system affect the latter part of the task. ${ }^{55}$ Thus, dopamine and 5-HT might be linked to different modes of decision making, with dopamine primarily involved in decision making under ambiguity and 5-HT in decision making under risk.

Recent evidence has indicated that the efficiency of the somatic marker system is influenced by genetic variations related to serotonergic and dopaminergic functioning. For example, Miu et $\mathrm{al}^{49}$ found that individuals homozygous for an allele associated with low efficiency of the serotonin transporter (5-HTT; the molecule involved in the synaptic reuptake of 5-HT) displayed better performance and stronger SCRs preceding disadvantageous selections on the IGT. This study indicates a facilitative effect of the allelic variation associated with the decreased reuptake of 5-HT. However, other studies of the association between 5-HTT alleles and IGT performance have yielded conflicting findings. ${ }^{56-59}$ With regards to the dopamine system, Roussos et al ${ }^{60}$ investigated the effects of allelic variations on the catechol-O-methyltransferase (COMT) gene on decision-making performance, and found an association between the allele conferring more efficient enzymatic degradation of catecholamines and adaptive decision making. This might seem inconsistent with the results from the study by Sevy et al ${ }^{54}$ mentioned earlier; however, enzymatic degradation of dopamine and dopamine reduction achieved by dietary challenge is not directly comparable. There is probably an optimal level of dopamine transmission associated with adaptive affective decision making.

Finally, the brain-derived neurotrophic factor (BDNF) gene has been linked to affective decision making. For example, Kang et $\mathrm{al}^{61}$ found an association between the Met allele on the $B D N F$ gene and reduced performance on the latter trials of the IGT. BDNF has been widely implicated in synaptic plasticity ${ }^{62}$ and might thus be involved in the encoding of option-outcome pairs. Consequently, the $B D N F$ gene might influence the efficiency of which emotional valence - ie, a somatic marker - is coupled with mental representations in neural circuits involved in somatic marker functions.

In sum, evidence indicates that 5-HT and dopamine play central and distinct roles in affective decision making. However, their precise roles remain unclear and genetic association studies into allelic variations that influence serotonergic activity have yielded conflicting findings that are not easily reconciled. Complex gene-environment and gene-by-gene interactions are likely to be involved. Thus, complex interactions between transmitter systems probably account for the final effect of somatic markers on decisional processes.

\section{Defective somatic marker signaling in addiction}

Both addicts and patients with orbitofrontal lesions display insensitivity to future consequences, difficulties in behavioral regulation, and impulse control deficits. ${ }^{7,32,53,63,64}$ The $\mathrm{SMH}$ has the potential to contribute to an understanding of these dysfunctional self-regulatory mechanisms in terms of the notion of an affective forecasting function that interacts with executive functions in behavioral selection. ${ }^{10}$ While defective somatic marker signaling could be involved in the maintenance of addictive behavior, the somatic marker model of addiction also assumes that addicts and nonaddicts differ in neural systems related to decision making and somatic state activation even before the addiction is established. ${ }^{7}$ Thus, in addition to biasing the individual to preserve maladaptive compulsive behavior, somatic marker defects are hypothesized to convey increased susceptibility to impulsive behavior and the development of addictive disorders. This diathetic assumptions imply that somatic marker defects 
are an endophenotypic biomarker for addictive behavior - eg, impulsion and compulsion.

\section{Imbalanced dual processing: the impulsive versus the reflective system}

The somatic marker model of addiction envisions addiction as a state characterized by an imbalance between an impulsive system that mediates the incentive motivational properties of emotionally competent stimuli, and a reflective system responsible for the control of impulses and the pursuit of longterm goals. ${ }^{65}$ This notion is compatible with the influential dual-process conceptualization of decision making. ${ }^{66-70}$

The impulsive system corresponds to neural circuits involved in appetitive approach behaviors. The amygdala and ventral striatum are believed to be integral structures in this system. For example, neurons in the ventral striatum are highly responsive to natural rewards, and in the case of addiction, they fire vigorously in response to the addictive object. ${ }^{71}$ Moreover, it has been shown that excitatory input from the basolateral amygdala to the nucleus accumbens determines the ensuing reward-seeking behavior in animal models. ${ }^{72,73}$ The impulsive system responds to emotionally competent stimuli with bioregulatory changes through its extensive connections with the subcortical effector structures. ${ }^{74}$ The ensuing changes bias the organism toward the rewarding stimulus - eg, a positive somatic marker is engaged for the stimulus - which may take on secondary inducer properties through the reflective system. The somatic marker model of addiction posits that the impulsive system may be dominant in addiction, a condition characterized by excessive emotional reactivity toward the addictive object.

The reflective system is associated with deliberate and goal-directed behavior, and is thought to correspond to regions of the prefrontal cortex and cingulate cortex. The system is dependent upon the functioning of neural circuits associated with cool executive functions like working memory and response inhibition, functions mediated primarily by the dorsolateral and ventrolateral sectors of the PFC, as well as hot executive functions like the secondary induction of somatic states and conflict resolution mediated by the medial PFC and anterior cingulate cortex. ${ }^{16,75}$ The operations of the reflective system are critical for decision making that is consistent with long-term prospects, and it is thought to be dysfunctional in disorders of addiction, rendering the system incapable of regulating the basic impulses associated with the addictive object. ${ }^{10}$
A neural structure gaining increased interest in recent years and considered to influence the efficiency of the two systems is the insular cortex. ${ }^{76,77}$ The insular cortex has been outlined as a structure capable of holding representations of somatic markers online to influence behavior and cognition, thus providing the basis for subjective feelings of interoceptive signals. ${ }^{18,36}$ The insular cortex may be involved in translating the homeostatic signals related to the condition of the body in withdrawal states into the subjective experience of craving. Indeed, a recent study of patients having suffered strokes in the insular region indicates that damage to this structure literally wipes out addiction to nicotine, a finding that implicates the insular cortex in the maintenance of addiction. ${ }^{78}$ The reduction in nicotine consumption is hypothesized to result from a failure in translating interoceptive information into conscious feelings, thus eliminating craving. Evidence indicates that the reduction of smoking behavior is even more pronounced when lesions include parts of the basal ganglia, thus damaging both homeostatic and impulsive circuitry. ${ }^{79}$ The insular cortex may serve a catalytic function for the impulsive system, amplifying the capacity of the addictive objects to trigger the system in withdrawal states. Moreover, insular projections may subvert or functionally hijack the reflective system in pursuit of the addictive object. ${ }^{80}$ This idea is compatible with the neuroanatomical evidence of the insular cortex's bidirectional connections to both the orbitofrontal cortex $(\mathrm{OFC})^{81}$ and the amygdala. ${ }^{82}$ Thus, a primarily insular-based homeostatic system could influence the dual systems in such a way that emotional signals initiated by these reward-driven circuits bias the addict toward the desired object.

\section{Neurophysiological abnormalities in addiction}

Neurophysiological irregularities found in addicts comprise areas involved in the somatic marker system, and which have been associated with dysfunctional decision making. Several abnormalities have been uncovered in regions corresponding to the reflective system in drug addiction. A consistent finding is reduced gray ${ }^{83}$ and white ${ }^{84}$ matter integrity and abnormal activation ${ }^{85}$ of the OFC. Decreased gray matter in the OFC has been found in various samples of drug addicts, including alcohol, ${ }^{86}$ heroin, ${ }^{87}$ cocaine,${ }^{88}$ methamphetamine, ${ }^{89}$ nicotine, ${ }^{90}$ and cannabinoid addiction. ${ }^{91}$ Furthermore, lower gray matter density has been reported in the $\mathrm{dlPFC}^{87}$ and the anterior cingulate cortex ${ }^{88,92,93}$ in various drug addictions, as compared to healthy controls. 
Similar findings have been uncovered in samples of behavioral addicts, although the evidence is mixed. For example, behavioral paradigms sensitive to orbitofrontal functioning (eg, the IGT) have indicated that the pathophysiology in the orbitofrontal/vmPFC region is related to pathological gambling. ${ }^{94}$ However, few studies have explored the structural correlates of pathological gambling, and those studies that have examined the morphological correlates of this disorder have failed to identify gross structural abnormalities in the OFC or associated regions of the frontal lobe. ${ }^{95}$ However, one study, in fact, found that a sample of pathological gamblers had increased gray matter density in the right OFC and the right ventral striatal area. ${ }^{96}$ Notably, it has also been found that pathological gamblers display increased functional connectivity between the right PFC and right ventral striatum. ${ }^{97}$ Studies of white matter integrity in pathological gambling have identified abnormalities that may affect frontal lobe functioning. ${ }^{98,99}$ These studies have demonstrated white matter microstructural abnormalities in the anterior corpus callosum, which contains tracts that are critical for signal transmission between the frontal hemispheres. These findings corroborate an early finding by Goldstein et al, ${ }^{100}$ suggesting that pathological gamblers displayed electroencephalographic activity indicating difficulties in shifting hemispheric activity in accordance with the shifts between tasks typically associated with either right or left hemispheric activation. Thus, abnormal communication between the different components of the reflective system (eg, the vmPFC, dIPFC, and anterior cingulate cortex) may be associated with dysfunction in this system in pathological gambling, which may result in a reduced ability to initiate prospective somatic markers to guide decisional processes toward long-term outcomes.

Unlike pathological gambling, studies into the morphological correlates of Internet addiction have uncovered widespread structural anomalies in the brain regions implicated in the reflective system. For example, various studies report volume reductions in the OFC, particularly in the right hemisphere. ${ }^{101-104}$ This hemispheric asymmetry is notable, as Bechara and Damasio ${ }^{16}$ indicated that somatic marker functions are somewhat lateralized to the right hemisphere. Furthermore, volume reductions have been reported in the bilateral dlPFC, ${ }^{104}$ left anterior cingulate cortex, ${ }^{104,105}$ and left posterior cingulate cortex ${ }^{105}$ in samples of Internet addicts. Moreover, studies employing structural imaging techniques have identified white matter abnormalities similar to those seen in drug addiction and pathological gambling. For example, a study by Lin et al ${ }^{106}$ revealed widespread white matter abnormalities in the OFC, anterior corpus callosum, cingulum, and the corona radiata. Similar findings were reported by Weng et al, ${ }^{103}$ who found extensive white matter reductions in the frontal lobe and the anterior corpus callosum. A recent study by Lin et a ${ }^{107}$ also found frontal lobe white matter deficits in a larger sample of Internet addicts, primarily located to the inferior frontal gyrus and the anterior cingulate cortex. These studies indicate that similar deficits in prefrontal cortical control systems may be shared by various addictions, and that these deficits may result in a pathological state characterized by increasing addiction-related behavior in the face of negative personal and social consequences due to a dysfunction in the system responsible for weighing the consequences of actions against their immediate benefit, and they may generate somatic states on the basis of these prospects.

In the sense of dual-process models on health behavior, ${ }^{108}$ a defective reflective system may leave the addict at the mercy of basic impulses generated by the impulsive system. These basic impulses may be triggered either by external (eg, drug cue, gambling cue, Internet cue) or internal (eg, thoughts or memories of the addictive object) stimuli. According to the somatic marker model of addiction, the trigger stimulus will cause a cascade of neural responses, some of which are involved in generating a somatic state that is relayed to structures involved in body mapping and homeostatic regulation (eg, insular cortex); these structures will then translate the somatic state information into a feeling (eg, urge or desire), which biases the addict toward the addictive object. ${ }^{10}$

Particularly, the ventral striatum and amygdala have emerged as important structures for the mediation of motivational properties of addiction-related stimuli. ${ }^{109,110}$ Indeed, neuroimaging studies have identified that these structures are consistently activated by cue-induction paradigms in addiction samples. ${ }^{11-113}$ In addition, it seems that addiction is characterized by a blunted reward circuitry response to nonaddiction cues pertaining to natural rewards like food and sex. ${ }^{114-116}$ The dominant view of this imbalanced processing of addiction versus nonaddiction cues is Robinson and Berridges' incentive sensitization theory, ${ }^{44,45}$ which posits that addiction is a result of abnormal salience attribution to addiction-related objects at the level of dopamine-innervated regions involved in the processing of reward (eg, the ventral striatum). Although the evidence for this position in the case of drug addiction seems to be convincing, it remains controversial whether it applies to nonchemical addictions. For example, recent studies have found that pathological gamblers do not display increased levels of dopamine release 
during the IGT. ${ }^{117,118}$ However, it was found that dopamine release was associated with decision-making deficits among pathological gamblers, in contrast to normal controls, for whom it was correlated with increased performance. ${ }^{118}$ This is notable, because it indicates that different neural processes are at play in the two groups, despite similar levels of dopamine release. It can be speculated that the addicted population displays poor performance because of a deficit in reflective system modulation of the impulsive system, which biases them against larger short-term rewards; even though these short-term rewards are associated with larger long-term losses. A recent neuroimaging study, however, identified that pathological gamblers had increased functional connectivity between the amygdala and ventral striatum during a value-based decision-making task. ${ }^{119}$ Increased functional connectivity between reward-related circuitry coupled with decreased functional connectivity between reflective circuits has been observed in samples of drug addicts. ${ }^{120}$ These findings support the notion of addiction as a state where addiction-related stimuli have an increased potential to elicit approach behavior through an impulsive system that is both overactive and unregulated.

The impulsive system is richly connected to effector structures at the level of the hypothalamus and brain stem. Through these connections, motivational objects have the capacity to change the somatic landscape. These changes are perceived by structures involved in body mapping and homeostatic regulation that give rise to conscious feelings of desire. Evidence indicates that the insular cortex is the main substrate in this process of somatic information translation. Recent interest in this structure in addiction research has resulted in evidence indicating maladaptive functioning of this system in addiction. ${ }^{77,121}$

Reduced gray matter volume has been reported in the insular cortex in both drug and behavioral addiction. For example, Franklin et al ${ }^{88}$ found decreased gray matter volume in the anterior insular cortex in a sample of cocaine addicts. Interestingly, these volume abnormalities did not correlate with the severity of the addiction, indicating that insula abnormalities may represent a premorbid vulnerability to addiction. Decreased gray matter volume in the insular cortex has also been reported for alcohol addicts. ${ }^{122}$ However, these volume reductions seem to be positively correlated with alcohol consumption, and preliminary evidence indicates that they reverse with abstinence. ${ }^{123}$ Although the few studies that have explored the structural correlates of pathological gambling have not reported morphological abnormalities in the insula, Internet addicts have been reported to display significant gray matter reductions in the insular region. ${ }^{103-105}$ One of these studies found that the reduction of volume correlated with scores on a measure of Internet addiction severity. ${ }^{103}$

Functional neuroimaging has shown that insular cortex activation to drug cues is increased in drug addicts relative to controls. ${ }^{124}$ Increased activity in the insular cortex has also been reported in pathological gamblers during exposure to gambling-related cues. ${ }^{112}$ Although increased insular activation would be predicted to occur among Internet addicts as well, studies of cue reactivity in this population have not observed increased insular activation to Internet stimuli. However, one study reported increased insular cortex activation at rest in Internet addiction. ${ }^{125}$ Some studies show that cue reactivity in the insular cortex is related to significant differences in clinical outcome variables in addiction. For example, Janes et al ${ }^{126}$ demonstrated that increased anterior insular cortex activation to smoking cues predicted slips among abstinent nicotine addicts, while Claus et al ${ }^{127}$ showed that increased insular activation was associated with addiction severity in alcohol addiction. Furthermore, Tsurumi et $\mathrm{a}^{128}$ found that insular activation in a reward anticipation task was inversely related to the duration of illness among abstinent pathological gamblers. While this finding seems somewhat at odds with the finding reported earlier, ${ }^{112}$ we suggest that this discrepancy may be due to the nature of the task. Tsurumi et al ${ }^{128}$ used points instead of money as the incentive and, thus, the decrease in activation may due to the specificity in the reward responses mentioned earlier. ${ }^{116}$

In sum, the findings summarized here indicate that addictions, both drug and behavioral, are characterized by neural abnormalities in various regions of the brain involved in somatic marker functioning. Both structural and functional abnormalities have been uncovered in the reflective, impulsive, and homeostatic system in addiction samples. Furthermore, there seems to be a substantial degree of overlap in neural abnormalities between various forms of addiction. This is in line with the diathetic assumption held by the somatic marker model, which states that addiction is characterized by neurocognitive deficits that exist a priori to the addiction as a vulnerability marker, and that these neurocognitive deficits are caused by abnormal functioning in the circuits that constitute the somatic marker system. However, the studies summarized here are cross-sectional; thus, causal conclusions are premature.

\section{Decision making in addiction}

As stated earlier, addiction is characterized by structural and functional abnormalities in regions implicated in cognitive 
and emotional processing. These abnormalities may manifest themselves behaviorally in domains involving both rational and emotional decision making. The somatic marker model of addiction holds that addiction is a state where the reflective system is compromised, which can result in a failure to utilize emotional marker signals pertaining to long-term outcomes in decisional processes, and thus the impulsive system, operating without the restraints of the reflective system, may bias the addict to immediate rewards. ${ }^{10}$ This section will review behavioral evidence pertinent to this account. The scope will be limited to studies involving the IGT, as this paradigm is believed to be sensitive to the cognitive defects arising from somatic marker failure. Indeed, neuroimaging studies have demonstrated that successful performance of the IGT is correlated with activation of neural architecture outlined by the $\mathrm{SMH} .{ }^{129}$

Studies employing the IGT in samples of drug addicts unequivocally demonstrate impaired affective decisionmaking performance..$^{22,130-133}$ This finding has emerged consistently across samples comprising various drug addict groups. Notably, Bechara and Damasio ${ }^{22}$ found that the maladaptive decision-making performance was associated with attenuated SCRs preceding disadvantageous card selections relative to controls, supporting the notion of somatic marker failure. However, it was also observed that a significant number of the addicts in the sample performed the task successfully. The decomposition of task performance into blocks reveals differences in learning curves, and has thus been the focus of research on addiction and decision making. Drug addicts display a shallower learning curve in comparison to controls; however, this also indicates that the drug addicts do not suffer from a general myopia of the future, as is the case for vmPFClesioned patients. ${ }^{22}$ Rather, this block-by-block analysis suggests that drug addicts are more driven by immediate outcomes, with a disproportional weighing of large rewards that could hamper learning of the task contingencies. In a follow-up study of the same sample, a reversed variant of the IGT was administered to test whether decreased performance was due to hyper-reactivity to reward or a general insensitivity to future consequences of actions. It was found that drug addicts could be subdivided into three groups according to their performance on the variant $\left.\mathrm{IGT}^{131}: 1\right)$ hyper-reactive to reward; 2) general insensitivity to future consequences; and 3) no impairment. This finding indicates an equifinality in the sense that addiction can develop from the failure of different components of the somatic system in unison or independently of each other. Reflective system deficits can hamper the ability to utilize prospective somatic markers pertinent to nega- tive future outcomes, while impulsive system hyper-reactivity can engender such a powerful approach-related signal that reflective processes are functionally overthrown. In line with this argument, Xiao et al $^{134}$ demonstrated that suboptimal IGT performance in a sample of adolescent binge drinkers was associated with increased activation in the left amygdala and bilateral insular cortex, as well as decreased OFC activation relative to controls.

The IGT has also been used to investigate decision making in pathological gambling, and the results are consistent with those obtained in substance addiction. ${ }^{135,136}$ However, few of these studies have included psychophysiological measurements. One exception is a study by Goudriaan et al, ${ }^{137}$ which found that affective decision-making defects were associated with attenuated SCRs preceding disadvantageous card selection on the IGT. This psychophysiological profile during IGT performance is consistent with that observed in the study by Bechara and Damasio, ${ }^{22}$ and lends further support to the notion of abnormal somatic marker functioning in pathological gambling. Moreover, a recent functional magnetic resonance imaging study by Power et al ${ }^{138}$ showed that impaired IGT performance in pathological gambling is associated with increased activation of the impulsive circuitry (amygdala and striatum), but also of the OFC.

Few studies have explored affective decision making in Internet addiction with the IGT. We identified four studies, ${ }^{139-142}$ and the results are mixed. While some studies have indicated that Internet addicts display decision-making deficits comparable to those reported in samples of drug addicts and pathological gamblers, ${ }^{139,140}$ others have indicated no impairment. ${ }^{141,142}$ However, the discrepancy in results may be, in part, due to the operational definitions of Internet addiction utilized. The study by Ko et $\mathrm{l}^{141}$ defined Internet addiction as Internet usage that surpasses 2 hours per day, while Metcalf and Pammer ${ }^{142}$ defined excessive Internet gaming (a form of Internet addiction) as 5 hours or more per week. Thus, it is likely that a substantial portion of the Internet addiction samples in these studies included participants that were not functionally impaired by their Internet use. It might be speculated that more rigorous inclusion criteria would yield results that are more representative of the affective decision-making capabilities that characterize Internet addicts.

The studies reviewed here have all relied on the IGT as a measure of affective decision making. However, it is notable that the task has received criticism. Foremost, the task's ability to capture affective decision-making capabilities has been challenged. Specifically, it has been argued that the task's reward/punishment schedule is cognitively 
penetrable, and thus task performance can be driven by conscious knowledge. ${ }^{143}$ In light of recent evidence, this criticism seems partially justified, as Guillaume et al ${ }^{144}$ demonstrated that differences in performance were related to conscious knowledge. However, task performance was also positively correlated with SCRs preceding disadvantageous card selections, and these were not associated with conscious knowledge. This suggests that both explicit knowledge and somatic markers contribute independently to task performance. However, it has been suggested that the interpretation of the SCRs as reflecting somatic marker biasing actions are incorrect, and there is some evidence supporting this suggestion. ${ }^{145}$ This represents a challenge for the SMH and epitomizes that it still is an evolving theoretical framework requiring empirical validation.

\section{The diathetic assumption: neurocognitive deficits as a predisposing factor}

A major tenet of the somatic marker model of addiction is that neurocognitive deficits related to abnormal somatic marker functioning is premorbid and acts as a predisposing factor to addiction. However, the study of this hypothesis is complicated by the fact that drugs of abuse have neurotoxic properties. ${ }^{146,147}$ Assuming that different forms of addiction have a similar pathophysiological and neurocognitive fundament, comparative studies of drug and behavioral addicts may dissociate the consequences of drug abuse from the neurocognitive deficits that are predisposing to addiction.

To dissociate the predisposing neurocognitive factors from drug-induced decrements in cognitive performance, Yan et $\mathrm{al}^{148}$ did a comparative study of heroin addicts and pathological gamblers where affective decision-making and working memory performance were tested. Their results indicated that affective decision-making defects are present in both disorders and are linked to years of abuse in heroin addiction, but not in pathological gambling. Working memory deficits were present only in heroin addiction. These results are similar to the ones obtained by Goudriaan et $\mathrm{al}^{149}$ in a comparison of alcohol addicts and pathological gambling. These studies suggest that affective decision-making capacities may represent a predisposing factor for addiction, and can be worsened and extended to other neurocognitive functions (eg, working memory) by the ingestion of substances with neurotoxic effects.

In support of the notion of affective decision-making capacity as a predisposing factor, Xiao et al ${ }^{150}$ showed that IGT score was a significant predictor of drinking behavior at a 1 year follow-up in a longitudinal study of Chinese adolescents. Similarly, IGT scores have been shown to be predictive of the development of smoking behaviors in an adolescent sample. ${ }^{151}$ These findings are corroborated by studies linking volumetric abnormalities in reflective circuits to future drug behavior. In one longitudinal study, it was found that smaller OFC volume at the age of 12 years predicted the initiation of cannabis use 4 years later. ${ }^{152}$ In a later publication, the same group reported that volumetric differences in the anterior cingulate cortex at the age of 12 years predicted problem drinking 4 years later. ${ }^{153}$ Moreover, Weiland et al ${ }^{154}$ found an inverse association between scores on an assessment of early risk for substance abuse and frontal cortex volume in young adults. Thus, while there is strong evidence for neurotoxic effects on neural circuitry associated with drug use, subtle neural abnormalities in regions involved in the somatic marker system may already be present prior to drug use. These abnormalities may be linked to distinct decisionmaking defects predisposing one toward the development of addictive behavior.

\section{Conclusion and future directions}

The goal of this paper has been to explore whether the $\mathrm{SMH}$ is applicable as a unifying explanatory framework of decision-making defects observed across different addictions, and whether the evidence supports somatic marker functioning as a predisposing factor for the development of addiction. The SMH is a neuroanatomical and neurocognitive decision-making framework that developed from studies of the decision-making defects that follow damage to the vmPFC. The impetus for the application of this framework in addiction research was the observation of comparable self-regulatory deficits between addicts and vmPFC patients, suggesting a common underlying mechanism. ${ }^{\text {? }}$

The SMH outlines a distinct neural architecture for its predictions, comprising a reflective system involved in selfregulatory functions permitting the pursuit of long-term goals and the weighing of consequences, an impulsive system that engenders motivational states in relation to emotionally salient stimuli, and a homeostatic system involved in adapting behavior to the condition of somatic systems. A predisposition to addiction could result from dysfunction in either one or a combination of these three systems. ${ }^{65}$ Indeed, there is substantial evidence that addiction to both substances and behaviors are characterized by morphological anomalies and abnormal activation patterns in the neural regions outlined by the SMH. ${ }^{83,85,88,93,98,102,111,112,119}$ Furthermore, preliminary evidence suggests that subtle abnormalities might predate the 
addiction as a diathesis for addiction development, ${ }^{152}$ and that these deficits can be accelerated by the use of psychoactive agents. ${ }^{148}$

The SMH states that the various neural components involved in the somatic marker system achieve adaptive decision making through an affective forecasting function that engenders somatic state activation in relation to option-outcome pairs. ${ }^{12}$ Studies of decision making in addiction have implied that addiction is characterized by a failure to engender appropriate somatic markers, which may represent a causal factor for the self-regulatory failures that characterize addiction. However, while dysfunctional decision making has consistently been observed in samples of drug and gambling addicts, ${ }^{22,135}$ the evidence regarding affective decision-making functions in Internet addiction is mixed. ${ }^{139-142}$ However, it is notable that the studies which did not find defective decision-making performance used inclusion criteria that are likely to result in a large number of participants that may not be regarded as true addicts. Future studies should aim to capture samples that are characterized by the core aspect of addiction - namely, persistent use despite increasing negative consequences. Moreover, previous studies have not investigated somatic state activation during task performance in Internet addiction. Thus, future studies could employ psychophysiological measures to investigate the notion of somatic marker failure in Internet addiction.

Both neuroanatomical abnormalities and decision-making defects have been shown to be predictive of substance use in adolescent samples. ${ }^{150,153}$ This implicates variations in somatic marker functioning as a predisposing factor, possibly implying that somatic marker defects could be an addiction endophenotype, promoting decision making that is both impulsive and compulsive. It follows from this suggestion that functional variations in the somatic marker system have a substantial genetic component, possibly related to genes encoding the efficiency of several interacting neurotransmitter systems. The serotonin system has been widely implicated in affective decision making, ${ }^{46,49,56}$ as well as in addiction development and maintenance. ${ }^{47,155}$ This suggests that genetic variations affecting the efficiency of the serotonergic system could be a central component in addiction risk through somatic marker failure. Longitudinal studies into the effects of polymorphisms implicated in serotonergic efficiency on decision-making capabilities and addiction proneness could be valuable in establishing the validity of this suggestion. Moreover, longitudinal studies could be instrumental in uncovering whether variations in somatic marker efficiency, operationalized as affective decision-making performance and somatic state activation, are related to different prognostic features in addiction populations.

In sum, the somatic marker model of addiction provides a plausible account of how emotion-related signals generated by immediate and future prospects can bias addicts toward addiction development and maintenance. Both neuroanatomical and behavioral predictions derived from the framework have the potential to further advance the current knowledge of how deficient decision making contributes to addiction. However, the model has some limitations. Foremost is an uncertainty of how to best test its predictions regarding decision-making performance. While the IGT has been the paradigm most associated with the framework, it has been criticized for being cognitively penetrable ${ }^{143}$ and possibly driven by other psychological mechanisms (eg, reversal learning ${ }^{156}$ ). Thus, it remains uncertain whether the IGT actually measures affective decision-making performance or some other construct. Furthermore, the interpretation of the psychophysiological changes (eg, SCR) preceding disadvantageous card selections on the task as reflecting somatic markers has been challenged. ${ }^{15}$ Therefore, an avenue for future research into the SMH in general and the somatic marker model of addiction would be to generate other paradigms that eliminate these uncertainties.

\section{Disclosure}

The authors report no conflicts of interest in this work.

\section{References}

1. Goodman A. Addiction: definition and implications. Br J Addict. 1990; 85(11):1403-1408.

2. Griffiths M. A 'components' model of addiction within a biopsychosocial framework. J Subst Use. 2005;10(4):191-197.

3. Brand M, Young KS, Laier C. Prefrontal control and internet addiction: a theoretical model and review of neuropsychological and neuroimaging findings. Front Hum Neurosci. 2014;8:375.

4. Conversano C, Marazziti D, Carmassi C, Baldini S, Barnabei G, Dell'Osso L. Pathological gambling: a systematic review of biochemical, neuroimaging, and neuropsychological findings. Harv Rev Psychiatry. 2012;20(3):130-148.

5. Potenza MN. Should addictive disorders include non-substance-related conditions? Addiction. 2006;101 Suppl 1:142-151.

6. Clark L, Robbins T. Decision-making deficits in drug addiction. Trends Cogn Sci. 2002;6(9):361.

7. Bechara A. Decision making, impulse control and loss of willpower to resist drugs: a neurocognitive perspective. Nat Neurosci. 2005;8(11): $1458-1463$.

8. Goldstein RZ, Volkow ND. Dysfunction of the prefrontal cortex in addiction: neuroimaging findings and clinical implications. Nat Rev Neurosci. 2011;12(11):652-669.

9. Limbrick-Oldfield EH, van Holst RJ, Clark L. Fronto-striatal dysregulation in drug addiction and pathological gambling: Consistent inconsistencies? Neuroimage Clin. 2013;2:385-393. 
10. Verdejo-García A, Bechara A. A somatic marker theory of addiction. Neuropharmacology. 2009;56 Suppl 1:48-62.

11. Bechara A, Damasio AR, Damasio H, Anderson SW. Insensitivity to future consequences following damage to human prefrontal cortex. Cognition. 1994;50(1-3):7-15.

12. Damasio A. Descartes'Error: Emotion, Reason, and the Human Brain. New York, NY: GP Putnam's Sons; 1994.

13. Bechara A, Tranel D, Damasio H, Damasio AR. Failure to respond autonomically to anticipated future outcomes following damage to prefrontal cortex. Cereb Cortex. 1996;6(2):215-225.

14. Damasio AR, Tranel D, Damasio H. Somatic markers and the guidance of behavior: theory and preliminary testing. In: Levin HS, Eisenberg HM, Benton AL, editors. Frontal Lobe Function and Dysfunction. Oxford, UK: Oxford University Press; 1991:217-229.

15. Dunn BD, Dalgleish T, Lawrence AD. The somatic marker hypothesis a critical evaluation. Neurosci Biobehav Rev. 2006;30(2):239-271.

16. Bechara A, Damasio AR. The somatic marker hypothesis: a neural theory of economic decision. Games Econ Behav. 2005;52(2):336-372.

17. Reimann M, Bechara A. The somatic marker framework as a neurological theory of decision-making: review, conceptual comparisons, and future neuroeconomics research. J Econ Psychol. 2010;31(5):767-776.

18. Zaki J, Davis JI, Ochsner KN. Overlapping activity in anterior insula during interoception and emotional experience. Neuroimage. 2012;62(1):493-499.

19. Bechara A, Damasio H, Tranel D, Damasio AR. Deciding advantageously before knowing the advantageous strategy. Science. 1997; 275(5304):1293-1295

20. Crone EA, Somsen RJ, Van Beek B, Van Der Molen MW. Heart rate and skin conductance analysis of antecendents and consequences of decision making. Psychophysiology. 2004;41(4):531-540.

21. Suzuki A, Hirota A, Takasawa N, Shigemasu K. Application of the somatic marker hypothesis to individual differences in decision making Biol Psychol. 2003;65(1):81-88.

22. Bechara A, Damasio H. Decision-making and addiction (part I) impaired activation of somatic states in substance dependent individuals when pondering decisions with negative future consequences. Neuropsychologia. 2002;40(10):1675-1689.

23. Gao Y, Raine A, Schug RA. Somatic aphasia: mismatch of body sensations with autonomic stress reactivity in psychopathy. Biol Psychol. 2012;90(3):228-233.

24. Schmitt WA, Brinkley CA, Newman JP. Testing Damasio's somatic marker hypothesis with psychopathic individuals: risk takers or risk averse? J Abnorm Psychol. 1999;108(3):538-543.

25. Miu AC, Heilman RM, Houser D. Anxiety impairs decision-making: psychophysiological evidence from an Iowa Gambling Task. Biol Psychol. 2008;77(3):353-358.

26. Cavedini P, Zorzi C, Baraldi C, et al. The somatic marker affecting decisional processes in obsessive-compulsive disorder. Cogn Neuropsychiatry. 2012;17(2):177-190.

27. Wölk J, Sütterlin S, Koch S, Vögele C, Schulz SM. Enhanced cardiac perception predicts impaired performance in the Iowa Gambling Task in patients with panic disorder. Brain Behav. 2014;4(2):238-246.

28. Brevers D, Noël X. Pathological gambling and the loss of willpower: a neurocognitive perspective. Socioaffect Neurosci Psychol. 2013;3:21592.

29. Bechara A. Risky business: emotion, decision-making, and addiction. J Gambl Stud. 2003;19(1):23-51.

30. Verdejo-García A, Pérez-García M, Bechara A. Emotion, decisionmaking and substance dependence: a somatic-marker model of addiction. Curr Neuropharmacol. 2006;4(1):17-31.

31. Damasio AR. Toward a neurobiology of emotion and feeling: operational concepts and hypotheses. Neuroscientist. 1995;1(1): 19-25.

32. Bechara A. The role of emotion in decision-making: evidence from neurological patients with orbitofrontal damage. Brain Cogn. 2004;55(1): 30-40.

33. Berlucchi G, Aglioti S. The body in the brain: neural bases of corporeal awareness. Trends Neurosci. 1997;20(12):560-564.
34. Craig AD. How do you feel - now? The anterior insula and human awareness. Nat Rev Neurosci. 2009;10(1):59-70.

35. Critchley HD, Rotshtein P, Nagai Y, O’Doherty J, Mathias CJ, Dolan RJ. Activity in the human brain predicting differential heart rate responses to emotional facial expressions. Neuroimage. 2005;24(3): 751-762.

36. Werner NS, Schweitzer N, Meindl T, Duschek S, Kambeitz J, Schandry R. Interoceptive awareness moderates neural activity during decision-making. Biol Psychol. 2013;94(3):498-506.

37. Sütterlin S, Schulz SM, Stumpf T, Pauli P, Vögele C. Enhanced cardiac perception is associated with increased susceptibility to framing effects Cogn Sci. 2013;37(5):922-935.

38. Clark L, Bechara A, Damasio H, Aitken MR, Sahakian BJ, Robbins TW. Differential effects of insular and ventromedial prefrontal cortex lesions on risky decision-making. Brain. 2008;131(Pt 5):1311-1322.

39. Weller JA, Levin IP, Shiv B, Bechara A. The effects of insula damage on decision-making for risky gains and losses. Soc Neurosci. 2009;4(4): 347-358.

40. Barbas H. Connections underlying the synthesis of cognition, memory, and emotion in primate prefrontal cortices. Brain Res Bull. 2000;52(5): 319-330.

41. Beckmann M, Johansen-Berg H, Rushworth MF. Connectivity-based parcellation of human cingulate cortex and its relation to functional specialization. J Neurosci. 2009;29(4):1175-1190.

42. Hinson JM, Jameson TL, Whitney P. Somatic markers, working memory, and decision making. Cogn Affect Behav Neurosci. 2002;2(4): $341-353$.

43. Jameson TL, Hinson JM, Whitney P. Components of working memory and somatic markers in decision making. Psychon Bull Rev. 2004;11(3): 515-520.

44. Robinson TE, Berridge $\mathrm{KC}$. The neural basis of drug craving: an incentive-sensitization theory of addiction. Brain Res Brain Res Rev. 1993;18(3):247-291.

45. Robinson TE, Berridge KC. Review. The incentive sensitization theory of addiction: some current issues. Philos Trans R Soc Lond B Biol Sci. 2008;363(1507):3137-3146.

46. Rogers RD. The roles of dopamine and serotonin in decision making: evidence from pharmacological experiments in humans. Neuropsychopharmacology. 2011;36(1):114-132.

47. Müller CP, Homberg JR. The role of serotonin in drug use and addiction. Behav Brain Res. 2015;277:146-192.

48. Homberg JR. Serotonin and decision making processes. Neurosci Biobehav Rev. 2012;36(1):218-236.

49. Miu AC, Crişan LG, Chiş A, Ungureanu L, Drug B, Vulturar R. Somatic markers mediate the effect of serotonin transporter gene polymorphisms on Iowa Gambling Task. Genes Brain Behav. 2012;11(4):398-403.

50. Rogers RD, Everitt BJ, Baldacchino A, et al. Dissociable deficits in the decision-making cognition of chronic amphetamine abusers, opiate abusers, patients with focal damage to prefrontal cortex, and tryptophandepleted normal volunteers: evidence for monoaminergic mechanisms. Neuropsychopharmacology. 1999;20(4):322-339.

51. Crockett MJ, Clark L, Lieberman MD, Tabibnia G, Robbins TW. Impulsive choice and altruistic punishment are correlated and increase in tandem with serotonin depletion. Emotion. 2010;10(6):855-862.

52. Schweighofer N, Bertin M, Shishida K, et al. Low-serotonin levels increase delayed reward discounting in humans. J Neurosci. 2008; 28(17):4528-4532.

53. Verdejo-García A, Lawrence AJ, Clark L. Impulsivity as a vulnerability marker for substance-use disorders: review of findings from high-risk research, problem gamblers and genetic association studies. Neurosci Biobehav Rev. 2008;32(4):777-810.

54. Sevy S, Hassoun Y, Bechara A, et al. Emotion-based decision-making in healthy subjects: short-term effects of reducing dopamine levels. Psychopharmacology (Berl). 2006;188(2):228-235.

55. Bechara A, Damasio H, Damasio AR. Manipulation of dopamine and serotonin causes different effects on covert and overt decision-making [abstract]. Society for Neuroscience Abstracts. 2001;27(1):1204. 
56. He Q, Xue G, Chen C, et al. Serotonin transporter gene-linked polymorphic region (5-HTTLPR) influences decision making under ambiguity and risk in a large Chinese sample. Neuropharmacology. 2010;59(6): $518-526$.

57. van den Bos R, Homberg J, Gijsbers E, den Heijer E, Cuppen E. The effect of COMT Val158 Met genotype on decision-making and preliminary findings on its interaction with the 5-HTTLPR in healthy females. Neuropharmacology. 2009;56(2):493-498.

58. Homberg JR, van den Bos R, den Heijer E, Suer R, Cuppen E. Serotonin transporter dosage modulates long-term decision-making in rat and human. Neuropharmacology. 2008;55(1):80-84.

59. Lage GM, Malloy-Diniz LF, Matos LO, Bastos MA, Abrantes SS, Corrêa $\mathrm{H}$. Impulsivity and the 5-HTTLPR polymorphism in a non-clinical sample. PLoS One. 2011;6(2):e16927.

60. Roussos P, Glakoumaki SG, Pavlakis S, Bitsios P. Planning, decisionmaking and the COMT rs4818 polymorphisms in healthy males. Neuropsychologia. 2008;46(2):757-763.

61. Kang JI, Namkoong K, Ha RY, Jhung K, Kim YT, Kim SJ. Influence of BDNF and COMT polymorphisms on emotional decision making. Neuropharmacology. 2010;58(7):1109-1113.

62. Lu B. BDNF and activity-dependent synaptic modulation. Learn Mem. 2003;10(2):86-98.

63. Berlin HA, Rolls ET, Kischka U. Impulsivity, time perception, emotion and reinforcement sensitivity in patients with orbitofrontal cortex lesions. Brain. 2004;127(Pt 5):1108-1126.

64. Morein-Zamir S, Robbins TW. Fronto-striatal circuits in responseinhibition: relevance to addiction. Brain Res. Epub September 16, 2014. pii: S0006-8993(14)01199-8.

65. Noël X, Brevers D, Bechara A. A neurocognitive approach to understanding the neurobiology of addiction. Curr Opin Neurobiol. 2013;23(4):632-638.

66. Bickel WK, Miller ML, Yi R, Kowal BP, Lindquist DM, Pitcock JA. Behavioral and neuroeconomics of drug addiction: competing neural systems and temporal discounting processes. Drug Alcohol Depend. 2007;90 Suppl 1:S85-S91.

67. Brocas I, Carrillo JD. Dual-process theories of decision-making: a selective survey. J Econ Psychol. 2014;41:45-54.

68. Evans JS. Dual-processing accounts of reasoning, judgment, and social cognition. Annu Rev Psychol. 2008;59:255-278.

69. Kahneman D, Tversky A. Prospect theory: an analysis of decision under risk. Econometrica. 1979;47:263-291.

70. Wiers RW, Stacy AW. Implicit cognition and addiction. Curr Dir Psychol Sci. 2006;15(6):292-296.

71. Kelley AE. Ventral striatal control of appetitive motivation: role in ingestive behavior and reward-related learning. Neurosci Biobehav Rev. 2004;27(8):765-776.

72. Ambroggi F, Ishikawa A, Fields HL, Nicola SM. Basolateral amygdala neurons facilitate reward-seeking behavior by exciting nucleus accumbens neurons. Neuron. 2008;59(4):648-661.

73. Stuber GD, Sparta DR, Stamatakis AM, et al. Excitatory transmission from the amygdala to nucleus accumbens facilitates reward seeking. Nature. 2011;475(7356):377-380.

74. Murray EA. The amygdala, reward and emotion. Trends Cogn Sci. 2007;11(11):489-497.

75. Noël X, Brevers D, Bechara A. A triadic neurocognitive approach to addiction for clinical interventions. Front Psychiatry. 2013;4:179.

76. Craig AD. Once an island, now the focus of attention. Brain Struct Funct. 2010;214(5-6):395-396.

77. Naqvi NH, Gaznick N, Tranel D, Bechara A. The insula: a critical neural substrate for craving and drug seeking under conflict and risk. Ann N Y Acad Sci. 2014;1316:53-70.

78. Naqvi NH, Rudrauf D, Damasio H, Bechara A. Damage to the insula disrupts addiction to cigarette smoking. Science. 2007;315(5811): 531-534.

79. Gaznick N, Tranel D, McNutt A, Bechara A. Basal ganglia plus insula damage yields stronger disruption of smoking addiction than basal ganglia damage alone. Nicotine Tob Res. 2014;16(4):445-453.
80. Verdejo-Garcia A, Clark L, Dunn BD. The role of interoception in addiction: a critical review. Neurosci Biobehav Rev. 2012;36(8): 1857-1869.

81. Ongür D, Price JL. The organization of networks within the orbital and medial prefrontal cortex of rats, monkeys and humans. Cereb Cortex. 2000;10(3):206-219.

82. Reynolds SM, Zahm DS. Specificity in the projections of prefrontal and insular cortex to ventral striatopallidum and the extended amygdala. J Neurosci. 2005;25(50):11757-11767.

83. Tanabe J, Tregellas JR, Dalwani M, et al. Medial orbitofrontal cortex gray matter is reduced in abstinent substance-dependent individuals. Biol Psychiatry. 2009;65(2):160-164.

84. Lim KO, Choi SJ, Pomara N, Wolkin A, Rotrosen JP. Reduced frontal white matter integrity in cocaine dependence: a controlled diffusion tensor imaging study. Biol Psychiatry. 2002;51(11):890-895.

85. London ED, Ernst M, Grant S, Bonson K, Weinstein A. Orbitofrontal cortex and human drug abuse: functional imaging. Cereb Cortex. 2000;10(3):334-342.

86. Durazzo TC, Tosun D, Buckley S, et al. Cortical thickness, surface area, and volume of the brain reward system in alcohol dependence: relationships to relapse and extended abstinence. Alcohol Clin Exp Res. 2011;35(6):1187-1200.

87. Liu H, Hao Y, Kaneko Y, et al. Frontal and cingulate gray matter volume reduction in heroin dependence: optimized voxel-based morphometry. Psychiatry Clin Neurosci. 2009;63(4):563-568.

88. Franklin TR, Acton PD, Maldjian JA, et al. Decreased gray matter concentration in the insular, orbitofrontal, cingulate, and temporal cortices of cocaine patients. Biol Psychiatry. 2002;51(2):134-142.

89. Kim SJ, Lyoo IK, Hwang J, et al. Prefrontal grey-matter changes in short-term and long-term abstinent methamphetamine abusers. Int $J$ Neuropsychopharmacol. 2006;9(2):221-228.

90. Kühn S, Schubert F, Gallinat J. Reduced thickness of medial orbitofrontal cortex in smokers. Biol Psychiatry. 2010;68(11):1061-1065.

91. Churchwell JC, Lopez-Larson M, Yurgelun-Todd DA. Altered frontal cortical volume and decision making in adolescent cannabis users. Front Psychol. 2010;1:225.

92. Matochik JA, London ED, Eldreth DA, Cadet JL, Bolla KI. Frontal cortical tissue composition in abstinent cocaine abusers: a magnetic resonance imaging study. Neuroimage. 2003;19(3):1095-1102.

93. Moreno-López L, Catena A, Fernández-Serrano MJ, et al. Trait impulsivity and prefrontal gray matter reductions in cocaine dependent individuals. Drug Alcohol Depend. 2012;125(3):208-214.

94. Cavedini P, Riboldi G, Keller R, D’Annucci A, Bellodi L. Frontal lobe dysfunction in pathological gambling patients. Biol Psychiatry. 2002;51(4):334-341.

95. van Holst RJ, de Ruiter MB, van den Brink W, Veltman DJ, Goudriaan AE. A voxel-based morphometry study comparing problem gamblers, alcohol abusers, and healthy controls. Drug Alcohol Depend. 2012;124(1-2):142-148.

96. Koehler S, Hasselmann E, Wüstenberg T, HeinzA, Romanczuk-Seiferth N. Higher volume of ventral striatum and right prefrontal cortex in pathological gambling. Brain Struct Funct. 2015;220(1):469-477.

97. Koehler S, Ovadia-Caro S, van der Meer E, et al. Increased functional connectivity between prefrontal cortex and reward system in pathological gambling. PLoS One. 2013;8(12):e84565.

98. Joutsa J, Saunavaara J, Parkkola R, Niemelä S, Kaasinen V. Extensive abnormality of brain white matter integrity in pathological gambling. Psychiatry Res. 2011;194(3):340-346.

99. Yip SW, Lacadie C, Xu J, et al. Reduced genual corpus callosal white matter integrity in pathological gambling and its relationship to alcohol abuse or dependence. World J Biol Psychiatry. 2013;14(2):129-138.

100. Goldstein L, Manowitz P, Nora R, Swartzburg M, Carlton PL. Differential EEG activation and pathological gambling. Biol Psychiatry. 1985;20(11):1232-1234.

101. Hong SB, Kim JW, Choi EJ, et al. Reduced orbitofrontal cortical thickness in male adolescents with internet addiction. Behav Brain Funct. 2013;9:11. 
102. Kühn S, Gallinat J. Brains online: structural and functional correlates of habitual Internet use. Addict Biol. 2015;20(2):415-422.

103. Weng CB, Qian RB, Fu XM, et al. Gray matter and white matter abnormalities in online game addiction. Eur J Radiol. 2013;82(8):1308-1312.

104. Yuan K, Cheng P, Dong T, et al. Cortical thickness abnormalities in late adolescence with online gaming addiction. PLoS One. 2013;8(1):e53055.

105. Zhou Y, Lin FC, Du YS, et al. Gray matter abnormalities in Internet addiction: a voxel-based morphometry study. Eur J Radiol. 2011;79(1):92-95.

106. Lin F, Zhou Y, Du Y, et al. Abnormal white matter integrity in adolescents with internet addiction disorder: a tract-based spatial statistics study. PLoS One. 2012;7(1):e30253.

107. Lin X, Dong G, Wang Q, Du X. Abnormal gray matter and white matter volume in 'Internet gaming addicts'. Addict Behav. 2015;40:137-143.

108. Hofmann W, Friese M, Strack F. Impulse and self-control from a dualsystems perspective. Perspect Psychol Sci. 2009;4(2):162-176.

109. Everitt BJ, Parkinson JA, Olmstead MC, Arroyo M, Robledo P, Robbins TW. Associative processes in addiction and reward. The role of amygdala-ventral striatal subsystems. Ann NY Acad Sci. 1999;877: 412-438.

110. Stamatakis AM, Sparta DR, Jennings JH, McElligott ZA, Decot H, Stuber GD. Amygdala and bed nucleus of the stria terminalis circuitry: Implications for addiction-related behaviors. Neuropharmacology. 2014;76 Pt B:320-328.

111. Chase HW, Eickhoff SB, Laird AR, Hogarth L. The neural basis of drug stimulus processing and craving: an activation likelihood estimation meta-analysis. Biol Psychiatry. 2011;70(8):785-793.

112. Goudriaan AE, de Ruiter MB, van den Brink W, Oosterlaan J, Veltman DJ. Brain activation patterns associated with cue reactivity and craving in abstinent problem gamblers, heavy smokers and healthy controls: an fMRI study. Addict Biol. 2010;15(4):491-503.

113. Ko CH, Liu GC, Hsiao S, et al. Brain activities associated with gaming urge of online gaming addiction. J Psychiatr Res. 2009;43(7): 739-747.

114. Asensio S, Romero MJ, Palau C, et al. Altered neural response of the appetitive emotional system in cocaine addiction: an fMRI Study. Addict Biol. 2010;15(4):504-516.

115. Kim JE, Son JW, Choi WH, et al. Neural responses to various rewards and feedback in the brains of adolescent Internet addicts detected by functional magnetic resonance imaging. Psychiatry Clin Neurosci. 2014;68(6):463-470.

116. Sescousse G, Barbalat G, Domenech P, Dreher JC. Imbalance in the sensitivity to different types of rewards in pathological gambling. Brain. 2013;136(Pt 8):2527-2538.

117. Linnet J, Møller A, Peterson E, Gjedde A, Doudet D. Dopamine release in ventral striatum during Iowa Gambling Task performance is associated with increased excitement levels in pathological gambling. Addiction. 2011;106(2):383-390.

118. Linnet J, Møller A, Peterson E, Gjedde A, Doudet D. Inverse association between dopaminergic neurotransmission and Iowa Gambling Task performance in pathological gamblers and healthy controls. Scand J Psychol. 2011;52(1):28-34.

119. Peters J, Miedl SF, Büchel C. Elevated functional connectivity in a striatal-amygdala circuit in pathological gamblers. PLoS One. 2013;8(9): 74353.

120. Ma N, Liu Y, Li N, et al. Addiction related alteration in resting-state brain connectivity. Neuroimage. 2010;49(1):738-744.

121. Naqvi NH, Bechara A. The insula and drug addiction: an interoceptive view of pleasure, urges, and decision-making. Brain Struct Funct. 2010;214(5-6):435-450.

122. Jung YC, Jang DP, Namkoong K, et al. Shape deformation of the insula in alcoholics: reduction of left-right asymmetry. Neuroreport. 2007;18(17):1787-1791.

123. Cardenas VA, Studholme C, Gazdzinski S, Durazzo TC, Meyerhoff DJ. Deformation-based morphometry of brain changes in alcohol dependence and abstinence. Neuroimage. 2007;34(3):879-887.
124. Jasinska AJ, Stein EA, Kaiser J, Naumer MJ, Yalachkov Y. Factors modulating neural reactivity to drug cues in addiction: a survey of human neuroimaging studies. Neurosci Biobehav Rev. 2014;38:1-16.

125. Park HS, Kim SH, Bang SA, Yoon EJ, Cho SS, Kim SE. Altered regional cerebral glucose metabolism in internet game overusers: a 18F-fluorodeoxyglucose positron emission tomography study. CNS Spectr. 2010;15(3):159-166.

126. Janes AC, Pizzagalli DA, Richardt S, et al. Brain reactivity to smoking cues prior to smoking cessation predicts ability to maintain tobacco abstinence. Biol Psychiatry. 2010;67(8):722-729.

127. Claus ED, Ewing SW, Filbey FM, Sabbineni A, Hutchison KE. Identifying neurobiological phenotypes associated with alcohol use disorder severity. Neuropsychopharmacology. 2011;36(10):2086-2096.

128. Tsurumi K, Kawada R, Yokoyama N, et al. Insular activation during reward anticipation reflects duration of illness in abstinent pathological gamblers. Front Psychol. 2014;5:1013.

129. Li X, Lu ZL, D’Argembeau A, Ng M, Bechara A. The Iowa Gambling Task in fMRI images. Hum Brain Mapp. 2010;31(3):410-423.

130. Bechara A, Dolan S, Denburg N, Hindes A, Anderson SW, Nathan PE. Decision-making deficits, linked to a dysfunctional ventromedial prefrontal cortex, revealed in alcohol and stimulant abusers. Neuropsychologia. 2001;39(4):376-389.

131. Bechara A, Dolan S, Hindes A. Decision-making and addiction (part II): myopia for the future or hypersensitivity to reward? Neuropsychologia. 2002;40(10):1690-1705.

132. Grant S, Contoreggi C, London ED. Drug abusers show impaired performance in a laboratory test of decision making. Neuropsychologia. 2000;38(8):1180-1187.

133. Whitlow CT, Liguori A, Livengood LB, et al. Long-term heavy marijuana users make costly decisions on a gambling task. Drug Alcohol Depend. 2004;76(1):107-111.

134. Xiao L, Bechara A, Gong Q, et al. Abnormal affective decision making revealed in adolescent binge drinkers using a functional magnetic resonance imaging study. Psychol Addict Behav. 2013;27(2):443-454.

135. Brevers D, Bechara A, Cleeremans A, Noël X. Iowa Gambling Task (IGT): twenty years after - gambling disorder and IGT. Front Psychol. 2013;4:665.

136. Wiehler A, Peters J. Reward-based decision making in pathological gambling: the roles of risk and delay. Neurosci Res. 2015;90: 3-14.

137. Goudriaan AE, Oosterlaan J, de Beurs E, van den Brink W. Psychophysiological determinants and concomitants of deficient decision making in pathological gamblers. Drug Alcohol Depend. 2006;84(3):231-239.

138. Power Y, Goodyear B, Crockford D. Neural correlates of pathological gamblers preference for immediate rewards during the iowa gambling task: an fMRI study. J Gambl Stud. 2012;28(4):623-636.

139. Sun DL, Chen ZJ, Ma N, Zhang XC, Fu XM, Zhang DR. Decisionmaking and prepotent response inhibition functions in excessive internet users. CNS Spectr. 2009;14(2):75-81.

140. Xu SH. Internet addicts' behavior impulsivity: evidence from the Iowa Gambling Task. Acta Psychologica Sinica. 2012;44:1523-1534.

141. Ko CH, Hsiao S, Liu GC, Yen JY, Yang MJ, Yen CF. The characteristics of decision making, potential to take risks, and personality of college students with Internet addiction. Psychiatry Res. 2010;175(1-2):121-125.

142. Metcalf O, Pammer K. Impulsivity and related neuropsychological features in regular and addictive first person shooter gaming. Cyberpsychol Behav Soc Netw. 2014;17(3):147-152.

143. Maia TV, McClelland JL. A reexamination of the evidence for the somatic marker hypothesis: what participants really know in the Iowa gambling task. Proc Natl Acad Sci U SA. 2004;101(45):16075-16080.

144. Guillaume S, Jollant F, Jaussent I, Lawrence N, Malafosse A, Courtet P. Somatic markers and explicit knowledge are both involved in decision-making. Neuropsychologia. 2009;47(10):2120-2124.

145. Tomb I, Hauser M, Deldin P, Caramazza A. Do somatic markers mediate decisions on the gambling task? Nat Neurosci. 2002;5(11): 1103-1104; author reply 1104. 
146. Angelucci F, Ricci V, Pomponi M, et al. Chronic heroin and cocaine abuse is associated with decreased serum concentrations of the nerve growth factor and brain-derived neurotrophic factor. J Psychopharmacol. 2007;21(8):820-825.

147. Zeigler DW, Wang CC, Yoast RA, et al; Council on Scientific Affairs, American Medical Association. The neurocognitive effects of alcohol on adolescents and college students. Prev Med. 2005;40(1): 23-32.

148. Yan WS, Li YH, Xiao L, Zhu N, Bechara A, Sui N. Working memory and affective decision-making in addiction: a neurocognitive comparison between heroin addicts, pathological gamblers and healthy controls. Drug Alcohol Depend. 2014;134:194-200.

149. Goudriaan AE, Oosterlaan J, de Beurs E, van den Brink W. Decision making in pathological gambling: a comparison between pathological gamblers, alcohol dependents, persons with Tourette syndrome, and normal controls. Brain Res Cogn Brain Res. 2005;23(1): $137-151$

150. Xiao L, Bechara A, Grenard LJ, et al. Affective decision-making predictive of Chinese adolescent drinking behaviors. J Int Neuropsychol Soc. 2009;15(4):547-557.
151. Xiao L, Koritzky G, Johnson CA, Bechara A. The cognitive processes underlying affective decision-making predicting adolescent smoking behaviors in a longitudinal study. Front Psychol. 2013;4:685.

152. Cheetham A, Allen NB, Whittle S, Simmons JG, Yücel M, Lubman DI. Orbitofrontal volumes in early adolescence predict initiation of cannabis use: a 4-year longitudinal and prospective study. Biol Psychiatry. 2012;71(8):684-692.

153. Cheetham A, Allen NB, Whittle S, Simmons J, Yücel M, Lubman DI. Volumetric differences in the anterior cingulate cortex prospectively predict alcohol-related problems in adolescence. Psychopharmacology (Berl). 2014;231(8):1731-1742.

154. Weiland BJ, Korycinski ST, Soules M, Zubieta JK, Zucker RA, Heitzeg MM. Substance abuse risk in emerging adults associated with smaller frontal gray matter volumes and higher externalizing behaviors. Drug Alcohol Depend. 2014;137:68-75.

155. Goldman D, Oroszi G, Ducci F. The genetics of addictions: uncovering the genes. Nat Rev Genet. 2005;6(7):521-532.

156. Fellows LK, Farah MJ. Different underlying impairments in decisionmaking following ventromedial and dorsolateral frontal lobe damage in humans. Cereb Cortex. 2005;15(1):58-63.
Psychology Research and Behavior Management

\section{Publish your work in this journal}

Psychology Research and Behavior Management is an international, peerreviewed, open access journal focusing on the science of psychology and its application in behavior management to develop improved outcomes in the clinical, educational, sports and business arenas. Specific topics covered include: Neuroscience, memory \& decision making; Behavior

\section{Dovepress}

modification \& management; Clinical applications; Business \& sports performance management; Social and developmental studies; Animal studies. The manuscript management system is completely online and includes a quick and fair peer-review system. Visit http://www.dovepress. com/testimonials.php to read real quotes from published authors. 